\title{
Hollow Cathode Magnetron Deposition of AlN thin Films: Crystalline Structure and Morphology
}

\author{
R. S. Pessoa, G. Murakami, G. Petraconi, H. S. Maciel, I. C. Oliveira, and K. G. Grigorov \\ Plasma and Processes Laboratory (LPP) - Department of Physics, \\ Technological Institute of Aeronautics, 12228-900, ITA - CTA, São José dos Campos-SP, Brazil
}

Received on 4 April, 2005

\begin{abstract}
A new dc hollow cathode plasma source has been assembled whith a conventional planar magnetron cathode used together with another plane cathode plate to form a hollow cathode cavity. The system comprises two cathode plates of aluminium separated by a distance $\mathrm{d}$, one of them acting as target of the magnetron cathode, the other being an ordinary plate. The discharge anode is a metallic flange of the vacuum chamber. This leads to enhanced ionization in the cathode cavity region and enables the discharge to operate at significantly lower pressures than for a typical planar magnetron configuration. As a consequence, sputtered atoms can reach a substrate with minimum energy loss due to collisions with filling gas atoms. The discharge gas was a mixture of argon and nitrogen. AlN thin films were grown on silicon substrates, at ambient temperature, and characterized with respect to the structure and morphology by XRD and AFM analyses respectively. The structure and roughness of the AlN films were studied as a function of the deposition parameters.
\end{abstract}

Keywords: Hollow cathode magnetron deposition; AlN thin films, XRD amd AFM

\section{INTRODUCTION}

Recently, aluminium nitrite (AlN) film has attracted substantial attention as a promising candidate for semiconductor material, electronic material for thermal dissipation, dielectric and passivation layer, surface acoustic wave (SAW) devices and photoelectric devices $[1,2]$. Many techniques, such as sputtering, chemical vapour deposition and laser chemical vapour deposition, have been used to fabricate AlN thin films on various substrates. In most cases the deposition temperatures are quite high. High temperature deposition has the disadvantage of degradation of the substrate and of the AIN thin film during deposition due to thermal damage. Hence, deposition of AlN thin films at low temperatures has become increasingly important and valuable. Hollow cathode magnetron (HCM) sputtering technique is promising under circumstances where low temperature deposition and conformal coatings are needed $[3,4]$.

Among the sputtering methods, the deposition by conventional d.c. magnetron sputtering is nowadays one of the most popular techniques to deposit thin films. In the magnetronsputtering source, the ability to modify and control the plasma can be of great benefit, especially for the production of high quality, specialized coatings [5]. One method used to modify the plasma properties in these magnetized plasmas is to inject $\gamma$-electrons into the plasma from a secondary source, for instance, from a hollow cathode or hot filament [6,7]. This reduces the cathode potential needed to sustain the discharge and lowers the plasma temperature. With HCM system, one can gain independent control over the discharge voltage and current, and has allowed some works to achieve very lowpressure operation $(<0.01 \mathrm{~Pa})$ [5,7] but with high discharge currents and dense plasmas.

In this work, we seek to extend the studies of ref. [3], by modification of the hollow cathode sputtering apparatus for AlN deposition. To assure the process control and reproducibility it is necessary to know the intrinsic plasma parame- ters. This aim was accomplished by utilizing various diagnostic tools, especially electrostatic probes and mass spectrometry. AlN thin films grown on silicon substrates, at ambient temperature for a exposure time of $15 \mathrm{~min}$, were characterized with respect to the structure and morphology by X-ray diffraction and AFM analyses respectively. The structure and roughness of the AlN films were studied as function of the deposition parameters.

\section{EXPERIMENTAL SET UP}

The modified magnetron sputtering device, called the hollow cathode magnetron (HCM), has been developed by surrounding a circular planar sputtering magnetron (PSM) cathode with a hollow cathode structure (HCS), see Fig. 1. The magnetron source is a commercial $33 \mathrm{~mm}$ diameter circular device with rare-earth permanent magnets producing parallel magnetic fields of about $0.04 \mathrm{~T}$ at the surface of $2.0 \mathrm{~mm}$ thick aluminum targets used here. Due to the $\mathrm{E} \times \mathrm{B}$ drift the electron path forms a circle whose radial dimensions depend on the radial uniformity of the magnetic field and on the discharge current. The magnetron target was cooled by ambient temperature water to prevent target melting in high power operation. The d.c. HCM operates typically at voltages in the range $(250$ - 400) $\mathrm{V}$ with a discharge current of $(10-500) \mathrm{mA}$. The process pressure was maintained in the range of $(0.05-0.5)$ $\mathrm{Pa}$ and measured using a Baratron type manometer.

The experiments were carried out in a cylindrical, stainless steel vacuum chamber $160 \mathrm{~mm}$ in diameter and $300 \mathrm{~mm}$ in length. A diffusion pump of $1500 \mathrm{l} / \mathrm{sec}$ backed by a mechanical pump evacuated the chamber into a base pressure of less than $10^{-4} \mathrm{~Pa}$. The aluminum cathodes were sputtered in a mixture of argon and nitrogen gases (12 sccm total flow: 50\% Ar, $50 \% \mathrm{~N}_{2}$ ). The gas is fed directly into the main chamber through the back chamber wall as shown in Fig. 1(a).

A cylindrical tungsten Langmuir single probe (length 0.2 
a)

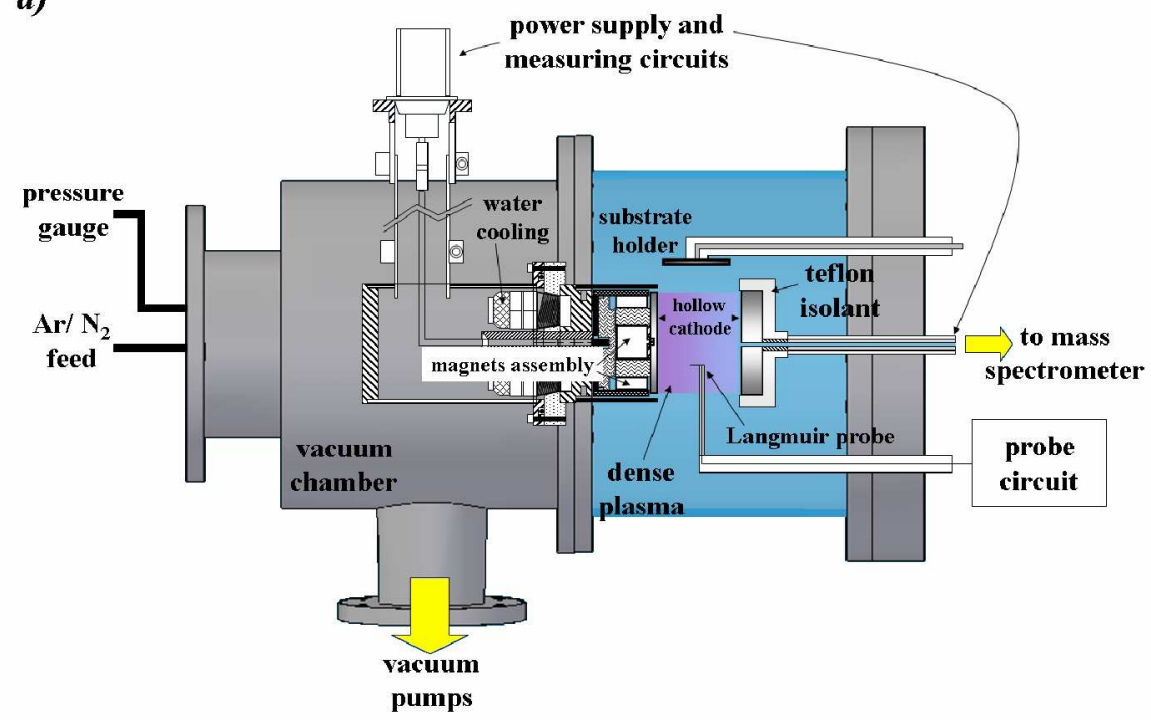

b)

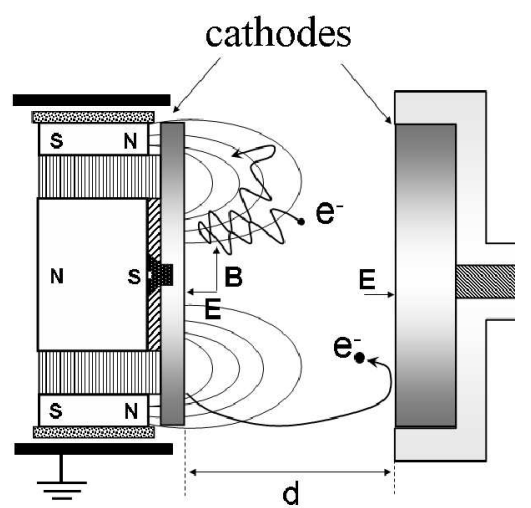

FIG. 1: a) Schematic diagram of the HCM source and diagnostic tools. b) Schema of the magnetron effect in the HCM. The high ExB near the target surface confines the emitted secondary electrons contributing so for more-efficient gas ionization.

$\mathrm{mm}$, diameter $0.1 \mathrm{~mm}$ ) was positioned along the discharge (Fig. 1).

The particles from the plasma are focused in direction to the mass spectrometer AccuQuad ${ }^{T M}$, with a resolution of 1 amu, through a drifting tube adapted to the vacuum chamber. The species were sampled through a micro orifice located at the mass spectrometer's entrance. The typical operation pressure within the mass spectrometer was $10^{-5} \mathrm{~Pa}$. The collected spectra were recorded in the mass range from 1 to $100 \mathrm{amu}$.

The type of crystalline structure, orientation and grain size was investigated by Philips 1830 diffractometer in $\theta-2 \theta$ scan mode. Moreover, the morphology of the surface and its roughness were studied using an Atom Force Microscope (AFM) SPM-9500 J3.

\section{RESULTS AND DISCUSSION}

\section{A. Discharge characteristics}

The HCM discharge properties were obtained taking into account the effect of the gas pressure and the inter-cathode distance. When the inter-cathode distance (d) is reduced, keeping the discharge voltage $\left(V_{d}\right)$ constant, the discharge current $\left(I_{d}\right)$ is seen to rise according to Figure 2. At optimized values of P.d, the discharge current is twice higher than in a conventional magnetron sputtering discharge.

Figure 2 shows that for inter-cathode distance between $(2.0$ - 3.0) $\mathrm{cm}$ the hollow cathode effects are enhanced. This allows discharge operation at low voltages and high discharge currents. The hollow cathode magnetron arrangement leads to the oscillatory motion of electrons between the cathodes sheaths and then to the more efficient ionization of the neutrals in the plasma bulk. This effect is enhanced at lower inter-

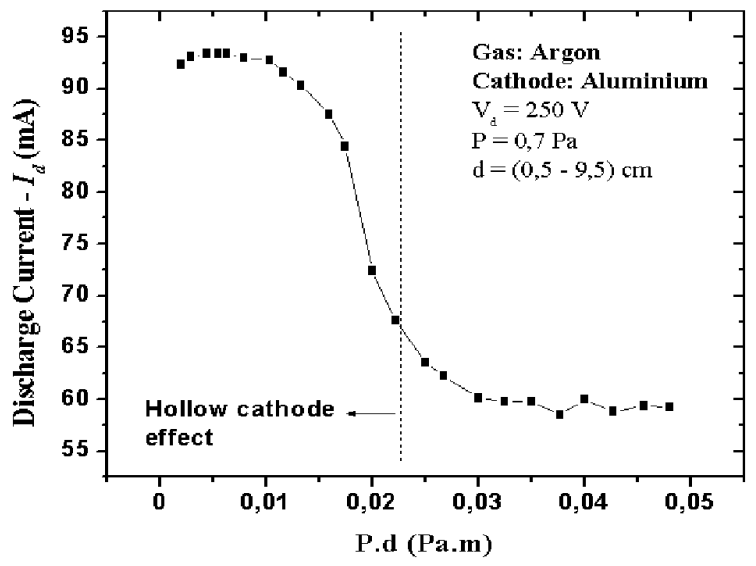

FIG. 2: Hollow Cathode discharge current as a function of the (P.d) parameter.

cathode distance and higher pressures. The physical reason of this condition is drawn by the fact that a sufficient number of collisions between electrons and molecules of the gas are required in the inter-cathodes space. At low pressure, when the mean free path is equal or greater than the inter-cathode distance, the electrons oscillate freely between the cathodes, thus reducing the discharge current (Fig.2).

The effect of the electrodes configuration on the magnetron discharge characteristic is show in the Fig. 3.

When HC configuration is considered, higher discharge current is obtained for the same gas pressure at any voltage compared to the usual electrodes configuration. Following the measured I-V discharge characteristics, displaced in Fig. 3, we can say that, much reduced cathode potential is required to maintain the same discharge current. For example, at a discharge current of $100 \mathrm{~mA}(\mathrm{P}=0.7 \mathrm{~Pa})$, the original diode 
magnetron operates at $320 \mathrm{~V}$, while the HCM configuration requires only $280 \mathrm{~V}$.

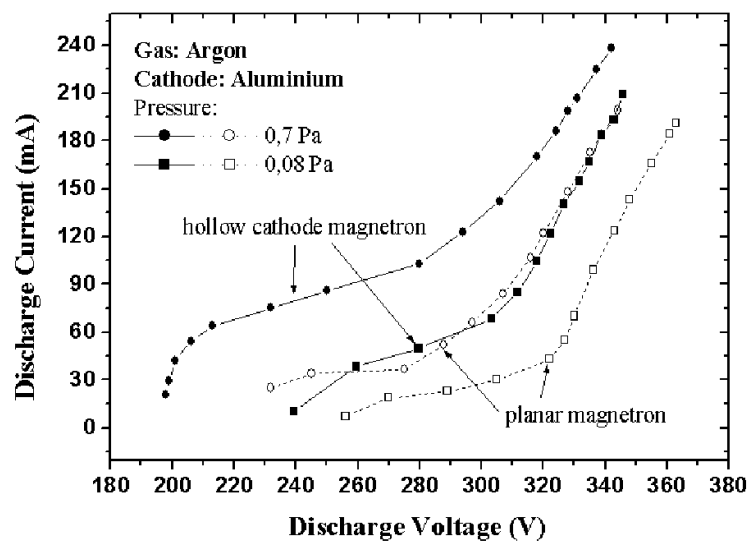

FIG. 3: Discharge I-V characteristics for both hollow cathode magnetron arrangement and conventional configuration.

\section{B. Analysis of plasma parameters of the HCM discharge}

Knowing the electron temperature and the plasma density nearby the cathode sheath is important in order to determine the positive ion flux towards the surface. Therefore, the temperature and the density of the electrons of the plasma in the vicinity of the substrate play an important role and determine in high degree the composition, the structure and the morphology of the growing films. Probe measurements were performed at gas pressure of $0.7 \mathrm{~Pa}$ and inter-cathode distance within the range of $(0.6-2.2) \mathrm{cm}$ using constant values of the discharge voltage and argon/nitrogen flow rate. The plasma has density of the order of $\left(10^{17}-10^{18}\right) \mathrm{m}^{-3}$ and electron temperature in the range of $(3-6) \mathrm{eV}$ (see Fig. 4).

Measures of the potential difference across the probe sheath immersed in the plasma helps to foresee with which energy the ion collides with a film or a substrate inserted in the plasma. Typical energy values are in the range $(15-26) \mathrm{eV}$.

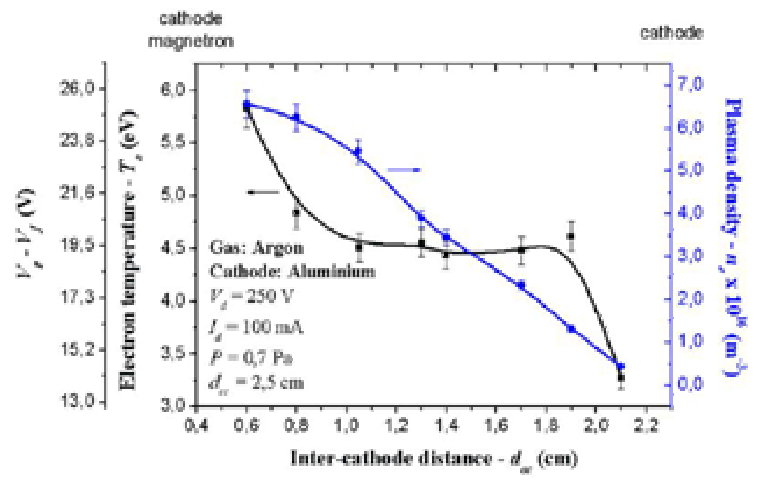

FIG. 4: Electron temperature and plasma density as a function of the inter-cathode distance.
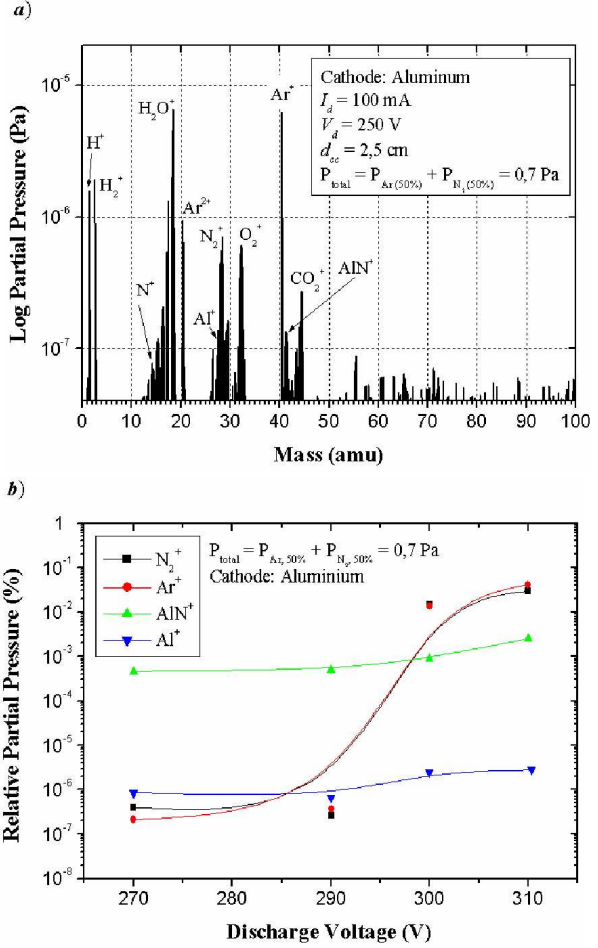

FIG. 5: (a) Mass spectrum for positive ions from $\mathrm{Al}$ sputtering. (b) Variation of the $\mathrm{Al}^{+}$and $\mathrm{AlN}^{+}$peaks with the discharge voltage at an $\mathrm{Ar} / \mathrm{N}_{2}$ pressure of $0.7 \mathrm{~Pa}$.

Figure 5(a) shows the positive ion mass spectrum during the reactive sputtering. The mass spectrum is densely packed, with the main contributions resulting from the process gas ions $\left(\mathrm{N}_{2}^{+}, \mathrm{Ar}^{+}\right)$plus their isotopes $\left({ }^{14} \mathrm{~N},{ }^{15} \mathrm{~N},{ }^{36} \mathrm{Ar},{ }^{38} \mathrm{Ar}\right.$, $\left.{ }^{40} \mathrm{Ar}\right)$, dissociation $\left(\mathrm{N}^{+}\right)$and double ionization $\left(\mathrm{Ar}^{2+}\right)$. Aluminum ions $\left(\mathrm{Al}^{+}\right)$are the most important, having their origin from the sputter target and corroborates to appearance of $\mathrm{AlN}^{+}$ion in the plasma medium. In Figure 5(b) the variation of the main species responsible for the formation of the $\mathrm{AlN}^{+}$ in function of the discharge power is shown.

\section{AIN-film analysis}

Figure 6 shows a typical AlN spectrum with very strong reflections at $32.88^{\circ}$ corresponding to AIN (100) crystalline orientations referring to the hexagonal type wurtzit. This sharp and intense peak is common for the highly textured phases similar to a single crystal. Besides the crystallographic orientation, another important parameter calculated is the coherent domain of reflection (CDR), which, in general, describes the dimensions of one crystal or grain containing micro crystals with identical spatial orientation of the axis $a, b$ and $c$. The CDR was calculated using the Debye-Scherrer formula revealing a columnar growth of crystals with dimension of $140 \mathrm{~nm}$-oriented with his axe "c" perpendicular to the silicon surface.

Figure 7 represents a typical AFM scan, with rms rough- 


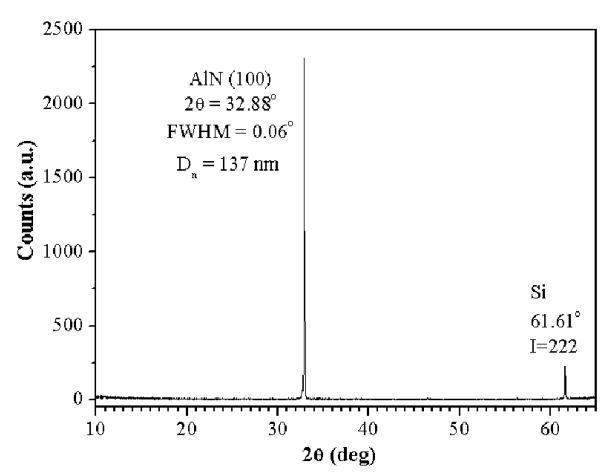

FIG. 6: X-ray diffraction pattern of sample deposited in $\mathrm{Ar}+\mathrm{N}_{2}$ atmosphere at $\mathrm{P}=0.7 \mathrm{~Pa}$ and $\mathrm{V}_{d}=250 \mathrm{~V}$.

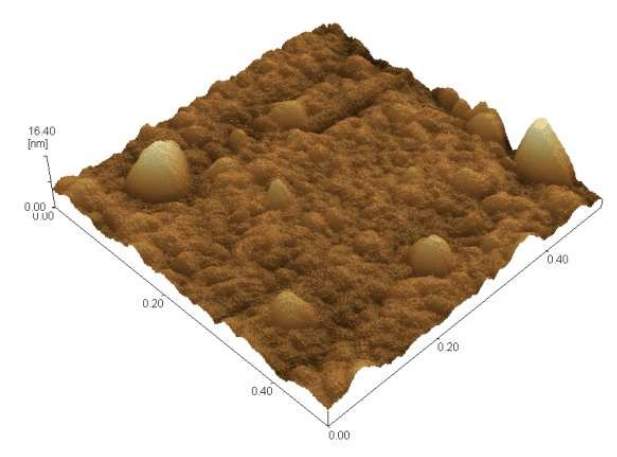

$500.00 \times 500.00[\mathrm{~nm}] \quad Z 0.00-16.40[\mathrm{~nm}]$

FIG. 7: AFM scan (dynamic mode) of AlN sample deposited in $\mathrm{Ar}+\mathrm{N}_{2}$ atmosphere for 15 minutes. ness below $2 \mathrm{~nm}$. The mean grain radius for this sample is 10 $\mathrm{nm}$ (as deduced from the statistic AFM surface engine) with standard deviation of $6 \mathrm{~nm}$.

\section{CONCLUSIONS}

In this work we studied a new - more efficient plasmas source combining a conventional planar magnetron with hollow-cathode system confining electrons and generating plasma densities in the order of $10^{17}-10^{18} \mathrm{~m}^{-3}$. The AlN thin films show distinct crystalline qualities growing in columns parallel to the substrate surface. This feature is especially interesting when SAW application is envisaged. Moreover the AFM study reveals a smooth and homogeneous surface, free of scratches and with roughness below $2 \mathrm{~nm}$. These material qualities are desirable for fabrication of optical filter. Another benefit of the new HCM source is its lowers deposition temperatures, which in conjunction with the off-axis deposition geometry allows films growth at room temperature. So, we expect that high quality AlN films could be grown with increased deposition rate onto polymer materials, which is an important requirement for the contemporary micro-and optoelectronic industry.

\section{Acknowledgement}

The financial support of CAPES is strongly acknowledged.
[1] H. Cheng, Y. Sun, J. X. Zhang, Y. B. Zhang, S. Yuan, and P. Hing, J. of Crystal Growth 254, 46 (2003) .

[2] M. A. Auger, L. Vazquez, M. Jergel, O. Sanchez, and J. M. Albella, Surf. and Coat. Technol. 180-181, 140 (2004).

[3] G. Petraconi, H. S. Maciel, and R. S. Pessoa, http://hal.ccsd.cnrs.fr/ccsd-00001921/en/, In: ICPP, Nice France (2004).

[4] C. J. Backhouse, S. K. Dew, and M. J. Brett, J. Vac. Sci. Technol. A 14(4), 2674 (1996).

[5] J. W. Bradley, D. M. Willett, and Y. A. Gonzalvo, J. Vac. Sci.
Technol. A 17(6), 3333 (1999).

[6] J. Musil, S. Kadlec, and W.D. Múnz, J. Vac. Sci. Technol. A9(3), 1171 (1991).

[7] J. J. Cuomo and S. M. Rossnagel, J. Vac. Sci. Technol. A4(3), 393 (1986).

[8] E. Klawuhn, G. C. D’Couto, K. A. Ashtiani, P. Rymer, M. A. Biberger, and K. B. Levy, J. Vac. Sci. Technol. A18(4), 1546 (2000). 